\title{
Research Article \\ On a New Integral Transform and Differential Equations
}

\author{
A. Kiliçman ${ }^{1}$ and H. Eltayeb ${ }^{2}$ \\ ${ }^{1}$ Department of Mathematics and Institute for Mathematical Research, Universiti Putra Malaysia, \\ 43400 UPM, Serdang, Selangor, Malaysia \\ ${ }^{2}$ Mathematics Department, College of Science, King Saud University, P.O. Box 2455, \\ Riyadh 11451, Saudi Arabia
}

Correspondence should be addressed to A. Kiliçman, akilicman@putra.upm.edu.my

Received 8 September 2009; Revised 5 April 2010; Accepted 6 May 2010

Academic Editor: Slimane Adjerid

Copyright (C 2010 A. Kiliçman and H. Eltayeb. This is an open access article distributed under the Creative Commons Attribution License, which permits unrestricted use, distribution, and reproduction in any medium, provided the original work is properly cited.

\begin{abstract}
Integral transform method is widely used to solve the several differential equations with the initial values or boundary conditions which are represented by integral equations. With this purpose, the Sumudu transform was introduced as a new integral transform by Watugala to solve some ordinary differential equations in control engineering. Later, it was proved that Sumudu transform has very special and useful properties. In this paper we study this interesting integral transform and its efficiency in solving the linear ordinary differential equations with constant and nonconstant coefficients as well as system of differential equations.
\end{abstract}

\section{Introduction}

In the literature there are numerous integral transforms and widely used in physics, astronomy as well as in engineering. In order to solve the differential equations, the integral transform were extensively used and thus there are several works on the theory and application of integral transform such as the Laplace, Fourier, Mellin, and Hankel, to name but a few. In the sequence of these transform, in early 90's Watugala [1] introduced a new integral transform, named the Sumudu transform and further applied it to the solution of ordinary differential equation in control engineering problems. For further detail and properties about Sumudu transform (see [2-7]) and many others. The Sumudu transform is defined over the set of the functions

$$
A=\left\{f(t): \exists M, \tau_{1}, \tau_{2}>0,|f(t)|<M e^{t / \tau_{j}}, \text { if } t \in(-1)^{j} \times[0, \infty)\right\},
$$


by the following formula

$$
G(u)=S[f(t) ; u]=: \int_{0}^{\infty} f(u t) e^{-t} d t, \quad u \in\left(-\tau_{1}, \tau_{2}\right) .
$$

The existence and the uniqueness was discussed in [5], for further details and properties of the Sumudu transform and its derivatives we refer to [2] and applied this new transform to solve the ordinary differential equations and control engineering problems, see $[1,8,9]$.

Further, in [3], some fundamental properties of the Sumudu transform were established. In [10], this new transform was applied to the one-dimensional neutron transport equation. In fact one can easily show that there is a strong relationship between double Sumudu and double Laplace transforms see, [5]. In [6], the Sumudu transform was extended to the distributions and some of their properties were studied.

In $[11,12]$, some further fundamental properties of the Sumudu transform were established. It turns out that the Sumudu transform has very special and useful properties and further it can help with intricate applications in sciences and engineering, see, for example, [10,13]. Once more, Watugala's work was followed by Weerakoon in [14, 15] by introducing a complex inversion formula for the Sumudu transform. In fact in [5], the existence and the uniqueness of Sumudu transform was discussed and some of the fundamental relationship established. In $[16,17]$, the further properties of Sumudu transform were studied and applied to the regular system of differential equations with convolution terms, respectively.

In this study, our purpose is to show the applicability of this interesting new transform and its efficiency in solving the linear ordinary differential equations with constant and non constant coefficients.

The existence and the uniqueness of Sumudu transform was discussed in [5] we reproduce as the next theorem that we will refer to it in the development of the present paper.

Theorem 1.1. If $f$ is of exponential order, then its Sumudu transform $S[f(t)]=F(u)$ is given by

$$
F(u)=\int_{0}^{\infty} e^{-t / u} f(t) d t
$$

where $1 / u=1 / \eta+i / \tau$. Then the integral exists in the right half plane $\eta>K$ and $\zeta>L$. Further, if $f(t)$ and $g(t)$ are having Sumudu transforms and given by $F(u)$ and $G(u)$, respectively, and if $F(u)=G(u)$ then $f(t)=g(t)$ where $u$ is a complex number.

Theorem 1.2. Let $f$ be Sumudu transformable and satisfy $f(t)=0$ for $t<0$. Then $\lim _{u \rightarrow \infty}(S[f](u))=0$.

Proof. Let $f_{0}(t)=f(t)[1-H(t-1)], f_{1}(t)=f(t) H(t-1)$. Since $f_{0}$ vanishes outside $[0,1]$, then we have $S[f](u)=\int_{0}^{1} e^{-t / u} f(t) d t$ for any $1 / u$. Moreover, $f=f_{0}+f_{1}, \operatorname{dom}\left(S\left(f_{1}\right)\right)=\operatorname{dom}(S(f))$ and $S[f](u)=\int_{0}^{1} e^{-t / u} f(t) d t+S\left(f_{1}\right)(u)$ for all $1 / u \in \operatorname{dom}(S(f))$. Let $1 / u_{0} \in \operatorname{dom}(S(f))$ and apply $|S(f)(u)| \leq A e^{-c / u}$ to $f_{1}$ we conclude that there is a constant $A$ such that

$$
|S(f)(u)| \leq \int_{0}^{1} e^{-t / u}|f(t)| d t+A e^{-1 / u} \quad \forall \frac{1}{u} \geq \frac{1}{u_{0}}
$$


as $1 / u \rightarrow \infty$ the second term on the right clearly tends to zero. The same applies to the first term.

Next we prove the following theorem that is very useful in the rest of this study.

Theorem 1.3. Let $\lambda>-1$. Then

(i) If $f \in \operatorname{loc}_{+}$and $\lim _{t \rightarrow \infty}\left[f(t) / t^{\lambda}\right]$ exists, so does $\lim _{1 / u \rightarrow 0+}\left[S[f(t)](u) / u^{\lambda+1}\right]$ and one has

$$
\lim _{t \rightarrow \infty}\left[\frac{f(t)}{t^{\lambda}}\right]=\frac{1}{\Gamma(\lambda+1)} \lim _{1 / u \rightarrow 0+}\left[\frac{S[f(t)](u)}{u^{\lambda+1}}\right] .
$$

(ii) if $f$ is Sumudu transformable and satisfies $f(t)=0$ for $t<0$ and if $\lim _{t \rightarrow 0+}\left[f(t) / t^{\lambda}\right]$ also $\lim _{1 / u \rightarrow \infty}\left[S[f(t)](u) / u^{\lambda+1}\right]$ and one has

$$
\lim _{t \rightarrow 0+}\left[\frac{f(t)}{t^{\lambda}}\right]=\frac{1}{\Gamma(\lambda+1)} \lim _{1 / u \rightarrow \infty}\left[\frac{S[f(t)](u)}{u^{\lambda+1}}\right] .
$$

Proof. (i) Let $f(t) / t \rightarrow \alpha$ as $t \rightarrow \infty$. This implies that there are constants $A$ and $\rho>0$ such that $|f(t)| / t^{\lambda} \leq A$ for $t>\rho$. This further implies that $e^{-t / u} f(t)$ is integrable for all $1 / u>0$ so that we may write, if $f(t)=0$ for $t<c$,

$$
S[f(t)](u)=\int_{c}^{\infty} e^{-t / u} f(t) d t=\int_{c}^{\rho} e^{-t / u} f(t) d t+\int_{\rho}^{\infty} e^{-t / u} f(t) d t
$$

now it is easy to see that $1 / u^{\lambda+1}$ time the first term on the right of (1.7) tends to zero as $1 / u \rightarrow 0^{+}$and $1 / u^{\lambda+1}$ times the second term on the right of (1.7) may be written for $1 / u>0$ as follows:

$$
\frac{1}{u^{\lambda}} \int_{\rho / u}^{\infty} e^{-x} f(u x) d x=\int_{\rho / u}^{\infty} x^{\lambda} e^{-x} \frac{f(u x)}{(u x)^{\lambda}} d x
$$

as $1 / u \rightarrow 0^{+}$and $f(u x) /(u x)^{\lambda}$ tends to $\alpha$ and, since it is bounded in the range of integration by the constant $A$. Then by the dominated convergence theorem and we conclude that

$$
\frac{1}{u^{\lambda+1}} S[f(t)](u) \longrightarrow \int_{\rho / u}^{\infty} x^{\lambda} e^{-x} \alpha d x=\alpha \Gamma(\lambda+1) \quad \text { as } \frac{1}{u} \longrightarrow 0^{+},
$$

which completes the proof.

(ii) Let $f(t) / t^{\lambda} \rightarrow \beta$ as $t \rightarrow 0^{+}$. Since this function is a bounded in the neighborhood of zero then there are constants $B$ and $\sigma>0$ such that $|f(t)| / t^{\lambda} \leq B$ for $0<t<\sigma$. Using a method similar to that in the proof of Theorem 1.3 we let $f_{0}=f(t)[1-H(t-\sigma)]$ and $f_{1}(t)=f(t) H(t-\sigma)$. Then

$$
S[f(t)](u)=\int_{0}^{\sigma} e^{-t / u} f(t) d t+S\left[f_{1}(t)\right](u) \quad \text { for } \frac{1}{u} \in \operatorname{dom} S[f]
$$


and apply $|S(f)(u)| \leq A e^{-c / u}$ we have $\left|S\left(f_{1}\right)(u)\right| \leq K e^{-\sigma / u}$ for some constant $K$ and $1 / u$ sufficiently large, and therefore $\left(1 / u^{\lambda+1}\right) S\left[f_{1}(t)\right](u) \rightarrow 0$ as $1 / u \rightarrow \infty$. Also, by a similar argument to that used in (i) then $1 / u^{\lambda+1}$ times the first term on the right of (1.10) tends to $\beta \Gamma(\lambda+1)$ as $1 / u \rightarrow \infty$, which completes the proof.

In the next proposition we prove the existence of Sumudu transform for the derivatives. In fact similar results were also reported, for example, in [10, 12, 13] by using different methods.

Proposition 1.4 (Sumudu transform of derivative). (i) Let $f$ be differentiable on $(0, \infty)$ and let $f(t)=0$ for $t<0$. Suppose that $f^{\prime} \in L_{\mathrm{loc}}$. Then $f^{\prime} \in L_{\mathrm{loc}} \operatorname{dom}(S f) \subset \operatorname{dom}\left(f^{\prime}\right)$ and

$$
S\left(f^{\prime}\right)=\frac{1}{u} S(f)-\frac{1}{u} f(0+) \quad \text { for } u \in \operatorname{dom}(S(f))
$$

(ii) More generally, if $f$ is differentiable on $(c, \infty), f(t)=0$ for $t<0$ and $f^{\prime} \in L_{\mathrm{loc}}$ then

$$
S\left(f^{\prime}\right)=\frac{1}{u} S(f)-\frac{1}{u} e^{-c / u} f(c+) \quad \text { for } u \in \operatorname{dom}(S(f)) .
$$

Proof. We start by (1.2) as follows, the local integrability of implies that $f(c+)$ exists, because, if $x>c$,

$$
f(x)=f(c+1)-\int_{x}^{c+1} f^{\prime}(t) d t \longrightarrow f(c+1)-\int_{c}^{c+1} f^{\prime}(t) d t \quad \text { as } x \longrightarrow c^{+} .
$$

Let $u \in \operatorname{dom}(S(f))$. If $w \in D_{0}=\{w: w$ differentiable and $w(0)=0\}$, integrating by part, we have

$$
\begin{aligned}
\frac{1}{u} \int \omega\left(\frac{t}{\lambda}\right) e^{-t / u} f^{\prime}(t) d t & =\frac{1}{u} \int_{c}^{\infty} \omega\left(\frac{t}{\lambda}\right) e^{-t / u} f^{\prime}(t) d t \\
& =\lim _{x \rightarrow c+}\left[-\frac{1}{u} \omega\left(\frac{x}{\lambda}\right) e^{-x / u} f(x)\right] \\
& -\frac{1}{u} \int_{c}^{\infty} e^{-t / u}\left[\frac{1}{\lambda} \omega^{\prime}\left(\frac{t}{\lambda}\right)-\frac{1}{u} \omega\left(\frac{t}{\lambda}\right)\right] f(t) d t
\end{aligned}
$$

Then we have

$$
\begin{aligned}
& -\frac{1}{u} \omega\left(\frac{c}{\lambda}\right) e^{-c / u} f(c+) \longrightarrow-\frac{1}{u} \omega(0) e^{-c / u} f(c+) \text { as } \lambda \longrightarrow \infty, \\
& -\frac{1}{u \lambda} \int e^{-t / u} \omega^{\prime}\left(\frac{t}{\lambda}\right) f(t) d t+\frac{1}{u^{2}} \int e^{-t / u} \omega\left(\frac{t}{\lambda}\right) f(t) d t, \quad \longrightarrow 0+\frac{1}{u} \omega(0) S(f)
\end{aligned}
$$


as $\mathcal{\lambda} \rightarrow \infty$ thus for any $\omega \in D_{0}$,

$$
\lim _{\lambda \rightarrow \infty}\left[\frac{1}{u} \int \omega\left(\frac{t}{\lambda}\right) e^{-t / u} f^{\prime}(t) d t\right]=\frac{\omega(0)}{u}[S(f)-f(c+)]
$$

This implies that $e^{-t / u} f^{\prime}(t)$ is convergent, that is, $u \in \operatorname{dom}(S(f))$, and that

$$
S\left(f^{\prime}\right)=\frac{1}{u} S(f)-\frac{1}{u} e^{-c / u} f(c+) .
$$

In general case, if $f$ is differentiable on $(a, b)$ with $a<b$, and $f(t)=0$ for $t<a$ or $t>b$ and $f^{\prime} \in L_{\text {loc }}$ then, for all $u$

$$
S\left(f^{\prime}\right)=\frac{1}{u} S(f)-\frac{1}{u} e^{-a / u} f(a+)+\frac{1}{u} e^{-b / u} f(b) .
$$

In the next example we show that Sumudu transform of a function can be obtained by using the differential equations.

Example 1.5. Let $y(t)=\sinh (\sqrt{t})$. Then

$$
\frac{d}{d t}\left[t y^{\prime}(t)\right]=\frac{1}{4} \sinh (\sqrt{t})+\frac{1}{4 \sqrt{t}} \cosh (\sqrt{t})=\frac{y(t)}{4}+\frac{y^{\prime}(t)}{2}, \quad t>0
$$

Now by taking Sumudu transform we have

$$
\frac{1}{u} S\left[t y^{\prime}(t)\right](u)-k=\frac{S[y(t)](u)}{4}+\frac{S\left[y^{\prime}(t)\right](u)}{2}
$$

where $k=\lim _{t \rightarrow 0+}\left[t f^{\prime}(t)\right]$. Then we have

$$
\frac{1}{u} S\left[t y^{\prime}(t)\right](u)=\frac{S[y(t)](u)}{4}+\frac{1}{2 u} S[y(t)](u)
$$

on noting that $f(0)=0$. Then it follows that

$$
16 u F^{\prime}(u)-(6-u) F(u)=0,
$$

and the solution given by

$$
F(u)=C^{\prime} e^{(1 / 4) u} \sqrt{u^{3}},
$$


replacing $u$ by $1 / s$ we obtain

$$
S[\sinh (\sqrt{t})](u)=\frac{\sqrt{\pi}}{2 s^{3 / 2}} e^{1 / 4 s}
$$

on noting that $\Gamma(3 / 2)=(1 / 2) \Gamma(1 / 2)=\sqrt{\pi} / 2$ and $\lim _{t \rightarrow 0+}(f(t) / \sqrt{t})=\lim _{t \rightarrow 0+}(\sinh \sqrt{t} / \sqrt{t})=$ 1 . This shows that the solution of some differential equations with non constant coefficients can be expressed as Sumudu transform.

In the next section we consider the Sumudu transform of higher derivatives and representation in the matrix form. However, first of all we introduce the following notation. Let $P(x)=\sum_{k=0}^{n}\left(a_{k} / x^{k}\right)$ be a polynomial in $1 / x$, where $n \geq 0$ and $a_{n} \neq 0$ then we define $M_{P}(x)$ to be the $1 \times n$ matrix given by

$$
M_{P}(x)=\left(\frac{1}{x} \frac{1}{x^{2}} \frac{1}{x^{3}} \cdots \frac{1}{x^{n-1}}\right)\left(\begin{array}{cccccc}
a_{1} & a_{2} & \cdot & \cdot & \cdot & a_{n} \\
a_{2} & a_{3} & \cdot & \cdot & a_{n} & 0 \\
a_{3} & \cdot & \cdot & a_{n} & 0 & 0 \\
\cdot & \cdot & \cdot & \cdot & \cdot & \cdot \\
a_{n} & 0 & \cdot & \cdot & \cdot & 0
\end{array}\right)
$$

Thus $M_{P}(x)$ defines a linear mapping of $\mathbb{C}^{n}$ into $\mathbb{C}$ in obvious way. We will write vectors $y$ in $\mathbb{C}^{n}$ as the row vectors or the column vectors interchangeably, whichever is convenient although, when $M_{P}(x) y$ is to be compute and the matrix representation by (1.25) of $M_{P}(x)$ is used, then of course $y$ must be written as a column vector

$$
M_{P}(x) y=\sum_{i=1}^{n} \frac{1}{x^{i}} \sum_{k=0}^{n-i} a_{i+k} y_{k}
$$

for any $y=\left(y_{0}, y_{1}, \ldots, y_{n-1}\right) \in \mathbb{C}^{n}$. If $n=0$, then $M_{P}(x)$ is a unique linear mapping of $\{0\}=\mathbb{C}^{0}$ into $\mathbb{C}$ (empty matrix). In general, if $n>0$ and $f$ is $n-1$ times differentiable on an interval $(a, b)$, with $a<b$, we will write

$$
\begin{aligned}
& \varphi(f ; a ; n)=\left(f(a+), f^{\prime}(a+), \ldots, f^{(n-1)}(a+)\right) \in \mathbb{C}^{n} \\
& \phi(f ; b ; n)=\left(f(b-), f^{\prime}(b-), \ldots, f^{(n-1)}(b-)\right) \in \mathbb{C}^{n} .
\end{aligned}
$$

If $a=0$, we write $\varphi(f ; n)$ for $\varphi(f ; 0 ; n)$. If $n=0$, we define

$$
\varphi(f ; a ; 0)=\phi(f ; a ; 0)=0 \in \mathbb{C}^{0} .
$$

Next we have the following proposition. 
Proposition 1.6 (Sumudu transform of higher derivatives). Let $f$ be $n$ times differentiable on $(0, \infty)$ and let $f(t)=0$ for $t<0$. Suppose that $f^{(n)} \in L_{\mathrm{loc}}$. Then $f^{(k)} \in L_{\mathrm{loc}}$ for $0 \leq k \leq n-$ $1, \operatorname{dom}(S f) \subset \operatorname{dom}\left(S f^{(n)}\right)$ and, for any polynomial $P$ of degree $n$,

$$
P(u) S(y)(u)=S(f)(u)+M_{P}(u) \varphi(y, n)
$$

for $u \in \operatorname{dom}(S f)$. In particular

$$
\left(S f^{(n)}\right)(u)=\frac{1}{u^{n}}(S f)(u)-\left(\frac{1}{u^{n}}, \frac{1}{u^{n-1}}, \ldots, \frac{1}{u}\right) \varphi(f ; n)
$$

(with $\varphi(f ; n)$ here written as a column vector). For $n=2$ one has

$$
\left(S f^{\prime \prime}\right)(u)=\frac{1}{u^{2}}(S f)(u)-\frac{1}{u^{2}} f(0+)-\frac{1}{u} f^{\prime}(0+) .
$$

Proof. We use induction on $n$. The result is trivially true if $n=0$, and the case $n=1$ is equivalent to the Proposition 1.4 (1.11). Now suppose that the result is true for some $n \geq 1$ and let $P(x)=\sum_{k=0}^{n+1}\left(a_{k} / x^{k}\right)$ having degree $n+1$ and writing in the form $P(x)=a_{0}+(1 / x) W(x)$, where $W(x)=\sum_{k=0}^{n}\left(a_{k+1} / x^{k}\right)$. Then it follows that $P(\dot{D}) f=a_{0} f+W(\dot{D}) z$ therefore by using Theorem 1.1 we have

$$
\begin{aligned}
S[P(\dot{D}) f](u)= & a_{0} S[f](u)+S[W(\dot{D}) z](u)-M_{W}(u) \varphi(z ; n) \\
= & a_{0} S[f](u)+W(u)\left[\frac{1}{u} S[f](u)-\frac{1}{u} f(0+)\right] \\
& -\sum_{i=1}^{n} \frac{1}{u^{i}} \sum_{k=0}^{n-i} a_{i+k+1} f^{(k+1)}(0+)
\end{aligned}
$$

on using (1.26) and setting $z^{(k)}=f^{(k+1)}$. Then the summation can be written in the form of

$$
\begin{aligned}
\sum_{i=1}^{n} \frac{1}{u^{i}} \sum_{k=1}^{n-i+1} a_{i+k} f^{(k)}(0+)= & \sum_{i=1}^{n+1} \frac{1}{u^{i}} \sum_{k=0}^{n-i+1} a_{i+k} f^{(k)}(0+) \\
& -\frac{1}{u}\left[\frac{1}{u^{n}} a_{n+1} f(0+)+\sum_{i=1}^{n} \frac{1}{u^{i-1}} a_{i} f(0+)\right] \\
& =M_{P}(u) \varphi(f ; n)-\frac{1}{u} W(u) f(0+) .
\end{aligned}
$$


Thus we have

$$
\begin{aligned}
S[P(\dot{D}) f](u)= & {\left[a_{0}+W(u) \frac{1}{u}\right] S[f](u)-\frac{1}{u} W(u) f(0+) } \\
& -M_{P}(u) \varphi(f ; n)+\frac{1}{u} W(u) f(0+) \\
= & P(u) S(f)(u)-M_{P}(u) \varphi(f ; n) .
\end{aligned}
$$

In general, if $f$ is differentiable on the open interval $(a, b)$, and $f(t)=0$ for $t<a$ or $t>b$ then $f^{(n)} \in L_{\mathrm{loc}}$ and

$$
S[P(\dot{D}) f](u)=P(u)(S f)(u)-M_{P}(u)\left[e^{-a / u} \varphi(f ; a ; n)-e^{-b / u} \phi(f ; b ; n)\right]
$$

for all $u$.

In particular case if we consider $y(t)=\sin (t)$ then clearly $y^{\prime \prime}+y=0$ and in the operator form we write

$$
\left(D^{2}+1\right) f=0
$$

Since $\operatorname{dom}(S f)$ contain $(0, \infty)$ then on using (1.29) and (1.42) with $n=2$ and $P(x)=x^{2}+1$, for $u>0$,

$$
0=\left(\frac{1}{u^{2}}+1\right) S(f)-\left(\frac{1}{u} \frac{1}{u^{2}}\right)\left(\begin{array}{ll}
0 & 1 \\
1 & 0
\end{array}\right)\left(\begin{array}{l}
0 \\
1
\end{array}\right)
$$

Since $\varphi(y, 2)=\left(f(0), f^{\prime}(0)\right)=(0,1)$. Thus we can obtain the same result without using definitions or transforms table as

$$
S[\sin (t) H(t)]=\frac{u}{u^{2}+1} .
$$

Now, in general form if we want to solve

$$
a_{n} y^{(n)}+a_{n-1} y^{(n-1)}+a_{n-2} y^{(n-2)} \cdots+a_{0}=f * g,
$$

then we rewrite in the form of

$$
P(\dot{D}) y=f * g,
$$

under the initial condition

$$
y(0)=y_{0}, \quad y^{\prime}(0)=y_{1}, \ldots, y^{(n-1)}(0)=y_{n-1},
$$


where $y$ is $n$ times differentiable on $(0, \infty)$, zero on $(-\infty, 0)$. Since $y^{(k)}$ is locally integrable therefore Sumudu transformable for $0 \leq k \leq n$ and, for every such $k$, then on using the Sumudu transform of (1.40) we have

$$
M_{P}(u) \varphi(y, n)=\left(\frac{1}{u} \frac{1}{u^{2}} \cdots \frac{1}{u^{n}}\right)\left(\begin{array}{cccccc}
a_{1} & a_{2} & \cdot & \cdot & \cdot & a_{n} \\
a_{2} & a_{3} & \cdot & \cdot & a_{n} & 0 \\
a_{3} & \cdot & \cdot & a_{n} & 0 & 0 \\
\cdot & \cdot & \cdot & \cdot & \cdot & \cdot \\
a_{n} & 0 & \cdot & \cdot & \cdot & 0
\end{array}\right)\left(\begin{array}{c}
y_{0} \\
y_{1} \\
\cdot \\
\cdot \\
\cdot \\
y_{n-1}
\end{array}\right)
$$

where $P(u)=a_{n} / u^{n}+a_{n-1} / u^{n-1}+\cdots+a_{0}$, and the nonhomogeneous term is single convolution. In particular, if $n=2$ we have

$$
\left(\frac{a_{2}}{u^{2}}+\frac{a_{1}}{u}+a_{0}\right) S(y)(u)=S(f * g)(u)+\left(\frac{1}{u} \frac{1}{u^{2}}\right)\left(\begin{array}{cc}
a_{1} & a_{2} \\
a_{2} & 0
\end{array}\right)\left(\begin{array}{l}
y_{0} \\
y_{1}
\end{array}\right)
$$

In order to get the solution of (1.40), we are taking inverse Sumudu transform for (1.29) as follows:

$$
y(t)=S^{-1}\left[\frac{(f * g)(u)}{P(u)}\right]+S^{-1}\left[\frac{M_{P}(u)}{P(u)} \phi(y, n)\right]
$$

provided that the inverse exist for each terms in the right-hand side of (1.44).

Now, multiply the right-hand side of (1.40) by polynomial $\Psi(t)=\sum_{k=0}^{n} t^{k}$, we obtain the following equation that is having non constant coefficients:

$$
\Psi(t) *[P(\dot{D}) y]=f * g,
$$

under the same initial conditions as above. By taking Sumudu transform for (1.45) and inverse Sumudu transform we have

$$
y=S^{-1}\left[\frac{F(u) G(u)}{k ! u^{k} P(u)}+\frac{1}{P(u)}\left(\frac{1}{u} \frac{1}{u^{2}} \cdots \frac{1}{u^{n+1}}\right)\left(\begin{array}{cccccc}
a_{1} & a_{2} & \cdot & \cdot & \cdot & a_{n} \\
a_{2} & a_{3} & \cdot & \cdot & a_{n} & 0 \\
a_{3} & \cdot & \cdot & a_{n} & 0 & 0 \\
\cdot & \cdot & \cdot & \cdot & \cdot & \cdot \\
a_{n} & 0 & \cdot & \cdot & \cdot & 0
\end{array}\right)\left(\begin{array}{c}
y_{0} \\
y_{1} \\
\cdot \\
\cdot \\
\cdot \\
y_{n-1}
\end{array}\right)\right],
$$

provided that the inverse transform exists. Now, if we substitute (1.46) into (1.45), we obtain the non homogeneous term of (1.45) $f * g$ and polynomial in the form of $\Phi(t)=$ $-\sum_{k=1}^{n}(1 / k !) t^{k}$. 
In particular consider the differential equation in the form of

$$
\begin{aligned}
& y^{\prime \prime \prime}-y^{\prime \prime}+4 y^{\prime}-4 y=e^{t} * \sin (t), \quad t>0, \\
& y(0)=1, \quad y^{\prime}(0)=4, \quad y^{\prime \prime}(0)=1 .
\end{aligned}
$$

On using (1.29) we have

$$
\begin{aligned}
& \left(\frac{1}{u^{3}}-\frac{1}{u^{2}}+\frac{4}{u}-4\right) S(y)(u)=u S\left[e^{t}\right] S[\sin (t)]+M_{P}(u) \varphi(y, 4) \\
& M_{P}(u) \varphi(y, 4)=\left(\frac{1}{u} \frac{1}{u^{2}} \frac{1}{u^{3}}\right)\left(\begin{array}{ccc}
4 & -1 & 1 \\
-1 & 1 & 0 \\
1 & 0 & 0
\end{array}\right)\left(\begin{array}{l}
1 \\
4 \\
1
\end{array}\right)=\frac{1}{u}+\frac{3}{u^{2}}+\frac{1}{u^{3}}
\end{aligned}
$$

after simplifying (1.48), we have

$$
Y(u)=\frac{u^{5}}{(1-u)\left(4 u^{2}+1\right)\left(1-u+4 u^{2}-4 u^{3}\right)}+\frac{\left(u^{2}+3 u+1\right)}{\left(1-u+4 u^{2}-4 u^{3}\right)} .
$$

By replacing the complex variables $u$ by $1 / s$ then (1.50) becomes

$$
Y\left(\frac{1}{s}\right)=\frac{s}{\left(s^{2}+4\right)\left(s^{2}+1\right)(s-1)^{2}}+\frac{s\left(s^{2}+3 s+1\right)}{\left(s^{2}+1\right)(s-1)} .
$$

To obtain the inverse Sumudu transform for (1.51) we use the following formula

$$
S^{-1}(Y(s))=\frac{1}{2 \pi i} \int_{\gamma-i \infty}^{\gamma+i \infty} e^{s t} Y\left(\frac{1}{s}\right) \frac{d s}{s}=\sum \text { residues }\left[e^{s t} \frac{Y(1 / s)}{s}\right],
$$

for more details see [18]. Then the solution of (1.47) given by

$$
y(t)=\frac{1}{6} \cos (t)+\frac{1}{10} e^{t} t+\frac{43}{50} e^{t}-\frac{2}{75} \cos (2 t)+\frac{38}{25} \sin (2 t) .
$$

Now if we multiply the right-hand side of (1.47) by $t^{2} *$ then the equation becomes

$$
\begin{gathered}
t^{2} *\left(y^{\prime \prime \prime}-y^{\prime \prime}+4 y^{\prime}-4 y\right)=2 \cos (2 t)-\sin (2 t), \quad t>0, \\
y(0)=1, \quad y^{\prime}(0)=4, \quad y^{\prime \prime}(0)=1,
\end{gathered}
$$

by applying similar method that we used above we obtain the solution of (1.54) in the form of

$$
y_{1}=\frac{1}{12} \sin (t)+\frac{27}{25} e^{t}+\frac{1}{20} e^{t} t-\frac{2}{25} \cos (2 t)+\frac{209}{150} \sin (2 t) .
$$


Now, if we substitute the solution of nonconstant coefficient in (1.54) we obtain the solution of the non homogeneous part of (1.54) plus the following terms $-(1 / 2) t^{2}$.

In fact the Sumudu transform is also applicable to the system of differential equations, see the details in [17].

Example 1.7. Solve for $t>0$ the system of two equations

$$
\begin{aligned}
& x^{\prime \prime}+2 y^{\prime}-2 x=-\sin (t), \quad x(0)=1, \quad x^{\prime}(0)=2, \\
& y^{\prime \prime}-2 x^{\prime}-2 y=\cos (t)-2, \quad y(0)=0, \quad y^{\prime}(0)=1 \text {. }
\end{aligned}
$$

The matrix

$$
P(u)=\left[\begin{array}{cc}
\frac{1}{u^{2}}-2 & \frac{2}{u} \\
-\frac{2}{u} & \frac{1}{u^{2}}-2
\end{array}\right]
$$

and we have $\operatorname{det}[P(u)]=1 / u^{4}+4$ which has degree $4=N(P)$. Thus $P$ regular. Now by applying Sumudu transform to the above system we have

$$
P(u) S\left(\begin{array}{l}
x \\
y
\end{array}\right)(u)=\left[\begin{array}{c}
-\frac{u}{u^{2}+1} \\
1+2 u^{2} \\
-\frac{u^{2}+1}{2}
\end{array}\right]+\Psi_{P}(u) \varphi(y, N(P))
$$

where $\Psi_{P}(u) \varphi(y, N(P))$ given by

$$
\Psi_{P}(u) \varphi(y, N(P))=\left[\begin{array}{cccc}
\frac{1}{u^{2}} & \frac{1}{u} & \frac{1}{u} & 0 \\
-\frac{1}{u} & 0 & \frac{1}{u^{2}} & \frac{1}{u}
\end{array}\right]\left[\begin{array}{l}
1 \\
2 \\
0 \\
1
\end{array}\right]=\left[\begin{array}{c}
\frac{1+2 u}{u^{2}} \\
-\frac{1}{u}
\end{array}\right]
$$

Then we obtain

$$
P(u)^{-1}=\left[\begin{array}{cc}
\frac{u^{2}\left(1-2 u^{2}\right)}{1+4 u^{4}} & \frac{-2 u^{3}}{1+4 u^{4}} \\
\frac{2 u^{3}}{1+4 u^{4}} & \frac{u^{2}\left(1-2 u^{2}\right)}{1+4 u^{4}}
\end{array}\right]
$$

Equation (1.58) becomes

$$
S\left(\begin{array}{l}
x \\
y
\end{array}\right)(u)=P^{-1}(u)\left[\begin{array}{c}
-\frac{u}{u^{2}+1} \\
1+2 u^{2} \\
-\frac{u^{2}+1}{2}
\end{array}\right]+P^{-1}(u)\left[\Psi_{P}(u) \varphi(y, N(P))\right]
$$


finally, by taking inverse Sumudu transform (1.61) we obtain the solution of the system as follows

$$
\begin{gathered}
x(t)=\sin (t)+e^{t} \cos (t), \\
y(t)=-\cos (t)+e^{t} \sin (t)+1,
\end{gathered}
$$

thus based on the above discussions we note that the Sumudu transform can be applied for system of differential equations thus can be used in many engineering problems. Similar applications can also be seen in $[5,10,11,13,19]$.

\section{Acknowledgments}

The authors gratefully acknowledge that this research was partially supported by the University Putra Malaysia under the Research University Grant Scheme 05-01-09-0720RU and Fundamental Research Grant Scheme 01-11-09-723FR. The authors also thank the referee(s) for very constructive comments and suggestions that improved the paper.

\section{References}

[1] G. K. Watugala, "Sumudu transform: a new integral transform to solve differential equations and control engineering problems," International Journal of Mathematical Education in Science and Technology, vol. 24, no. 1, pp. 35-43, 1993.

[2] M. A. Asiru, "Sumudu transform and the solution of integral equations of convolution type," International Journal of Mathematical Education in Science and Technology, vol. 32, no. 6, pp. 906-910, 2001.

[3] M. A. Asiru, "Further properties of the Sumudu transform and its applications," International Journal of Mathematical Education in Science and Technology, vol. 33, no. 3, pp. 441-449, 2002.

[4] M. A. Asiru, "Classroom note: application of the Sumudu transform to discrete dynamic systems," International Journal of Mathematical Education in Science and Technology, vol. 34, no. 6, pp. 944-949, 2003.

[5] F. B. M. Belgacem, A. A. Karaballi, and S. L. Kalla, "Analytical investigations of the Sumudu transform and applications to integral production equations," Mathematical Problems in Engineering, no. 3-4, pp. 103-118, 2003.

[6] H. Eltayeb, A. Kiliçman, and B. Fisher, "A new integral transform and associated distributions," Integral Transforms and Special Functions, vol. 21, no. 5, pp. 367-379, 2010.

[7] V. G. Gupta and B. Sharma, "Application of Sumudu transform in reaction-diffusion systems and nonlinear waves," Applied Mathematical Sciences, vol. 4, no. 9, pp. 435-446, 2010.

[8] G. K. Watugala, "Sumudu transform: a new integral transform to solve differential equations and control engineering problems," Mathematical Engineering in Industry, vol. 6, no. 4, pp. 319-329, 1998.

[9] G. K. Watugala, "The Sumudu transform for functions of two variables," Mathematical Engineering in Industry, vol. 8, no. 4, pp. 293-302, 2002.

[10] A. Kadem, "Solving the one-dimensional neutron transport equation using Chebyshev polynomials and the Sumudu transform," Analele Universitatii din Oradea. Fascicola Matematica, vol. 12, pp. 153-171, 2005.

[11] F. B. M. Belgacem, “Boundary value problem with indefinite weight and applications," International Journal of Problems of Nonlinear Analysis in Engineering Systems, vol. 10, no. 2, pp. 51-58, 1999.

[12] F. B. M. Belgacem and A. A. Karaballi, "Sumudu transform fundamental properties investigations and applications," Journal of Applied Mathematics and Stochastic Analysis, pp. 1-23, 2006.

[13] M. G. M. Hussain and F. B. M. Belgacem, "Transient solutions of Maxwell's equations based on sumudu transform," Progress in Electromagnetics Research, vol. 74, pp. 273-289, 2007.

[14] S. Weerakoon, "Complex inversion formula for Sumudu transform," International Journal of Mathematical Education in Science and Technology, vol. 29, no. 4, pp. 618-621, 1998. 
[15] S. Weerakoon, "Application of Sumudu transform to partial differential equations," International Journal of Mathematical Education in Science and Technology, vol. 25, no. 2, pp. 277-283, 1994.

[16] A. Kiliçman and H. Eltayeb, "On the applications of Laplace and Sumudu transforms," Journal of the Franklin Institute, vol. 347, no. 5, pp. 848-862, 2010.

[17] A. Kiliçman, H. Eltayeb, and P. R. Agarwal, "On Sumudu transform and system of differential equations," Abstract and Applied Analysis, vol. 2010, Article ID 598702, 11 pages, 2010.

[18] A. Kiliçman and H. E. Gadain, "An application of double Laplace transform and double Sumudu transform," Lobachevskii Journal of Mathematics, vol. 30, no. 3, pp. 214-223, 2009.

[19] I. Ali and S. L. Kalla, "A generalized Sumudu transform," Bulletin of Pure and Applied Mathematics, vol. 1, no. 2, pp. 192-199, 2007. 


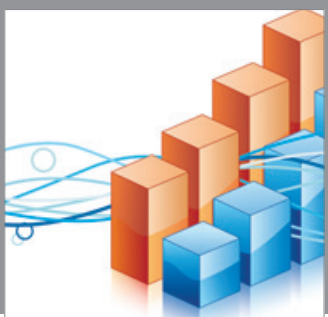

Advances in

Operations Research

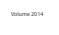

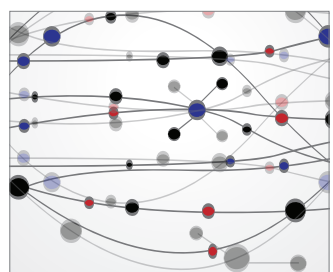

\section{The Scientific} World Journal
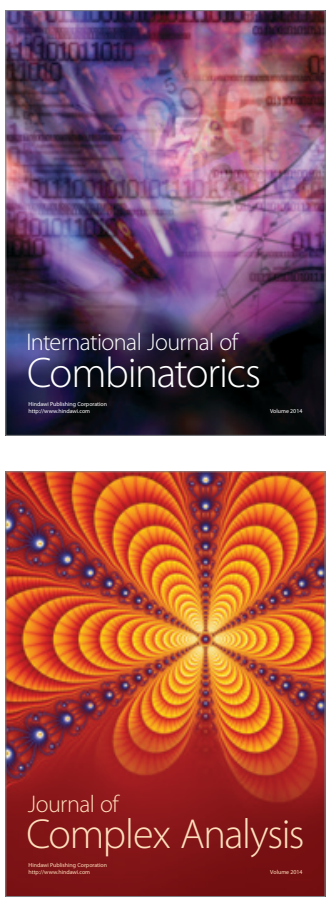

International Journal of

Mathematics and

Mathematical

Sciences
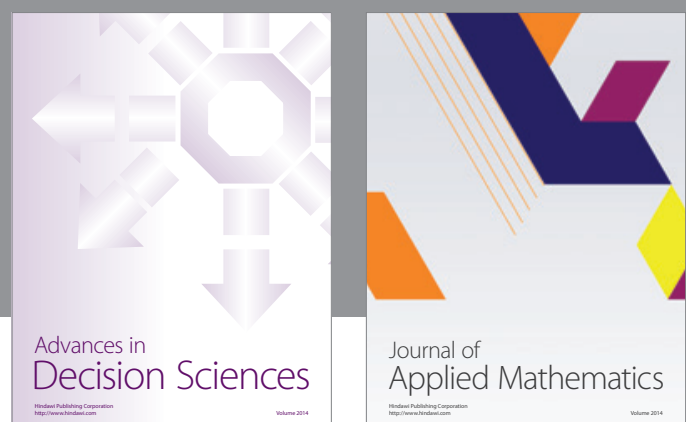

Journal of

Applied Mathematics
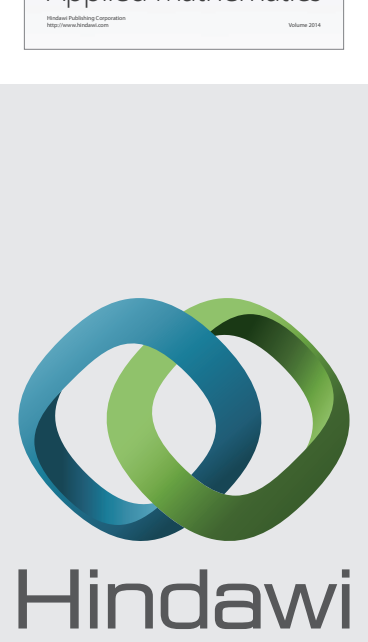

Submit your manuscripts at http://www.hindawi.com
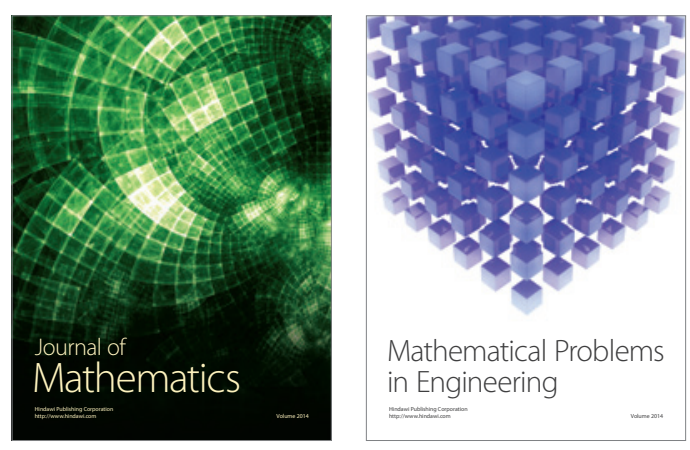

Mathematical Problems in Engineering
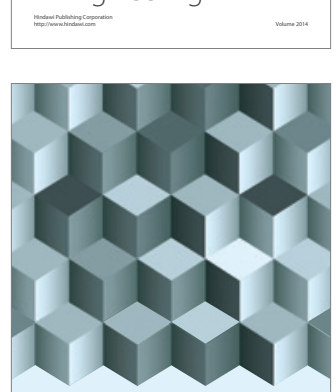

Journal of

Function Spaces
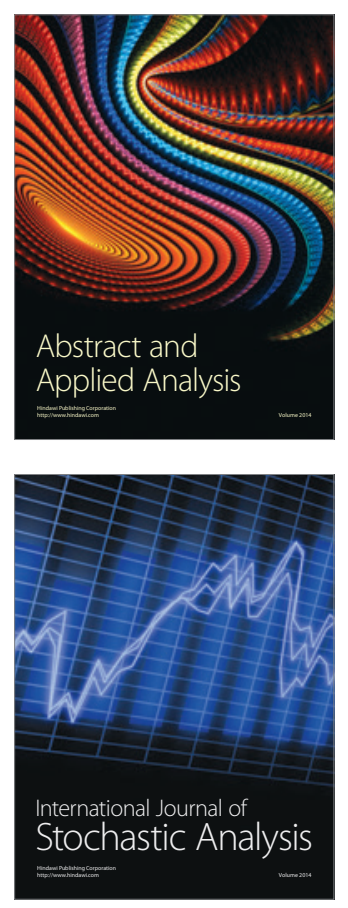

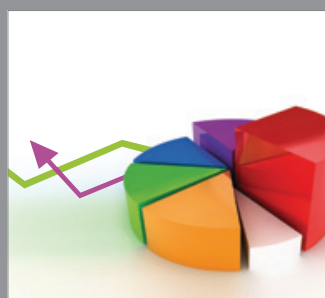

ournal of

Probability and Statistics

Promensencen
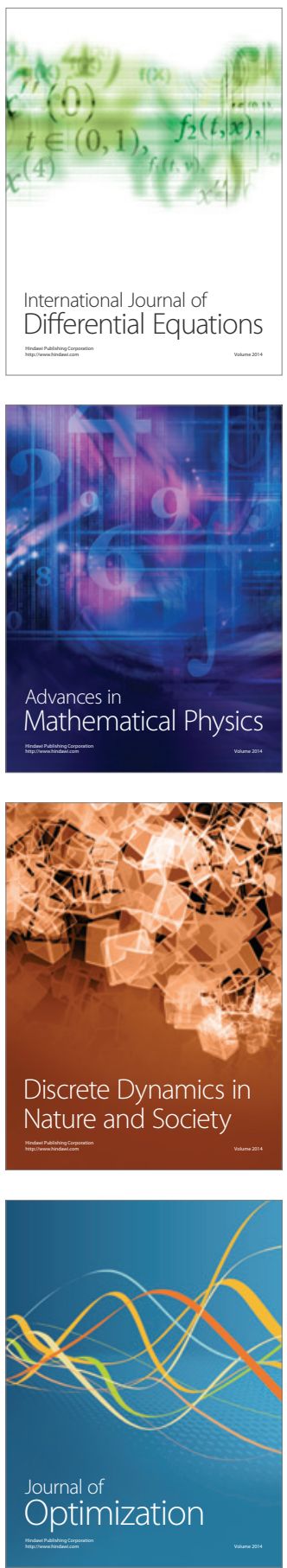